\title{
Abordagem ecofisiológica dos manguezais: uma revisão
}

\author{
Sávia Soares Pascoalini ${ }^{1}$ \\ Dielle Meire de Santana Lopes ${ }^{2}$ \\ Antelmo Ralph Falqueto ${ }^{2}$ \\ Mônica Maria Pereira Tognella ${ }^{2 *}$ \\ ${ }^{1}$ Universidade Federal do Espírito Santo, Vitória - ES, Brasil \\ ${ }^{2}$ Universidade Federal do Espírito Santo, Centro Universitário Norte do Espírito Santo \\ Rodovia BR 101 Norte, Km 60, CEP 29932-540, São Mateus - ES, Brasil \\ * Autor para correspondência \\ monica.tognella@gmail.com
}

Submetido em 30/11/2013

Aceito para publicação em 26/05/2014

\section{Resumo}

O manguezal apresenta elevada produtividade primária que é, em parte, resultado dos mecanismos fisiológicos aplicados pelas espécies vegetais às restrições ambientais. O objetivo desta síntese é avaliar o estado da arte dos estudos ecofisiológicos sobre os manguezais e identificar lacunas que possibilitem ampliar o conhecimento científico sobre os manguezais brasileiros e suas potenciais contribuições para as mudanças climáticas. $\mathrm{O}$ agravamento das restrições ambientais, como o aumento da salinidade, maior tempo de alagamento e deficiência de nutrientes, induz a diminuição da assimilação fotossintética, resultando na redução do desenvolvimento das espécies. A resposta de determinada espécie ao estresse depende de sua tolerância. Conclui-se que os estudos ecofisiológicos da vegetação de mangue são pontuais, e seus resultados divergem entre os estudos de campo e laboratório. No Brasil, esse conhecimento ainda é incipiente, dificultando a previsão do comportamento das espécies diante das mudanças climáticas.

Palavras-chave: Fotossíntese; Inundação; Nutrientes; Salinidade

\section{Abstract}

Ecophysiological approach to mangroves: a review. Mangrove has a high primary productivity that partly results from physiological mechanisms applied by plant species to environmental restrictions. This synthesis aims to assess the state of the art of ecophysiological studies on mangroves and identify gaps that allow increasing scientific knowledge on Brazilian mangroves and their potential contributions to climate changes. The worsening of environmental restrictions, such as increased salinity, longer flooding, and nutrient deficiency, induces a decrease of photosynthetic assimilation, resulting in a reduction in the development of species. The response of a given species to stress depends on its tolerance. We conclude that ecophysiological studies on mangrove vegetation are occasional, and their results differ between field and laboratory studies. In Brazil, this knowledge is still incipient, making it difficult to predict the behavior of species in face of climate change.

Key words: Flooding; Nutrients; Photosynthesis; Salinity 


\section{Introdução}

A presente revisão foi realizada com o intuito de avaliar o estado da arte dos estudos ecofisiológicos para os manguezais mundiais. Para isto, realizamos levantamento bibliográfico nos principais periódicos onde são publicados temas relacionados à ecologia deste ecossistema, bem como em revistas especializadas na fisiologia vegetal. Além disso, literatura clássica sobre o tema e publicada em livros especializados sobre o manguezal também subsidiam nossa síntese.

Os manguezais despertam interesse de especialistas na área de ecofisiologia vegetal pela particularidade de sua vegetação. Estas, de acordo com Tomlinson (1986), enfrentam condições de elevada salinidade e temperatura, ampla variação da maré (submetendoas à inundação), deficiência de nutrientes, sedimento inconsolidado, entre outros fatores físico-químicos. Este ambiente, com condições adversas daquelas enfrentadas pelas demais plantas terrestres (FARNSWORTH, 2000), só foi possível de ser colonizado devido aos diferentes mecanismos morfológicos, anatômicos, fisiológicos e bioquímicos apresentados pelas espécies vegetais, os quais, integrados, contribuem para sua elevada produtividade (POOP et al., 1985; BALL, 1988; NAIDOO et al., 2002; 2011; PARIDA et al., 2004; BARR et al., 2009).

Alterações na temperatura em função das mudanças climáticas podem ocasionar modificações nas áreas de distribuição e na diversidade atual dos manguezais. Estudos relacionados à obtenção de dados in situ de fotossíntese diária e sazonal são escassos e restritos a poucas espécies, o que dificulta previsões futuras sobre o comportamento das espécies perante as modificações climáticas.

Portanto, estes mecanismos fisiológicos empregados por essas espécies vegetais são o foco desta revisão, na qual abordaremos análises dos processos de fotossíntese em condições de baixa e elevada salinidade, inundação e deficiência de nutrientes. O objetivo é avaliar os estudos ecofisiológicos dos manguezais e identificar carências que permitam ampliar o conhecimento científico deste ecossistema no Brasil e suas potenciais contribuições para as mudanças climáticas.

\section{Fotossíntese}

As espécies de mangue são consideradas plantas $\mathrm{C}_{3}$, pois não apresentam anatomia de plantas $\mathrm{C}_{4}$, sua composição isotópica $\left(\delta^{13} \mathrm{C}\right)$ varia entre 24,6 e 32,2 \%o, seu ponto de compensação do $\mathrm{CO}_{2}$ e sua taxa máxima de fotossíntese em temperatura abaixo de $35^{\circ} \mathrm{C}$ são características consideradas típicas de plantas $\mathrm{C}_{3}$ (BALL; FARQUHAR, 1984a; 1984b; BALL, 1986; PARIDA et al., 2004). Os estudos pioneiros de Ball e Farquhar (1984a; 1984b) demonstram que apesar do declínio na assimilação com aumento da salinidade de 50 para $500 \mathrm{nM}$ de $\mathrm{NaCl}$, não ocorrem diferenças no ponto de compensação do $\mathrm{CO}_{2}$ característicos das $\mathrm{C}_{3}$.

A fotossíntese é o resultado conjunto de três processos: o processo fotoquímico, onde ocorre a absorção de fótons e produção de compostos reduzidos (ATP e NADPH) necessários ao processo bioquímico, que por sua vez é responsável pela assimilação fotossintética do carbono e também pelos processos de trocas gasosas. Estas tratam da entrada de $\mathrm{CO}_{2}$ para o interior da planta e o efluxo de $\mathrm{O}_{2}$ para a atmosfera através dos estômatos (SOBRADO, 2000; NAIDOO et al., 2002). Além da parte bioquímica, a assimilação fotossintética está sujeita à interferência das condições abióticas as quais as espécies vegetais estão submetidas e que podem resultar em limitação da produção primária (BALL, 1988; NAIDOO et al., 2002).

Ao estudar a eficiência fotoquímica de manguezais, Naidoo et al. (2002) observaram que espécies mais tolerantes à salinidade incrementam sua eficiência fotoquímica com o aumento da salinidade, uma vez que promovem maior assimilação de $\mathrm{CO}_{2}$ quando comparadas com as espécies não tolerantes. Sobrado (2000) também verificou o mesmo comportamento para Avicennia germinans (L.) L., Laguncularia racemosa (L.) Gaertn. e Rhizophora mangle L., sendo que as primeiras obtiveram maior assimilação de carbono do que $R$. mangle menos tolerante ao sal. $\mathrm{O}$ autor relaciona o maior desempenho fotossintético realizado por aquelas espécies ao melhor uso de água em nível foliar, apesar de apresentarem menor transporte de água. 


\section{Salinidade: seus efeitos sobre a planta}

A salinidade no ambiente marinho e no manguezal deve-se, principalmente, ao cloreto de sódio $(\mathrm{NaCl})$ dissolvido na água, correspondente a $86 \%$ do total. A concentração média desses sais na água do mar é de $35 \mathrm{~g} / \mathrm{kg}$, correspondendo a $483 \mathrm{mM}$ de $\mathrm{Na}^{+}$e $558 \mathrm{mM}$ de $\mathrm{Cl}^{-}$(LIBES, 2009). É considerado o principal fator abiótico ao qual as espécies do manguezal estão sujeitas (PARIDA; JHA, 2010). Dessa forma, diversos estudos em laboratório foram desenvolvidos para avaliar a capacidade de crescimento e/ou a germinação do mangue com relação à salinidade.

Estas espécies apresentam comportamento distinto em relação à salinidade. Por exemplo, Bruguiera parviflora (Roxb.) Wt. \& Arn. ex Griff. tem seu máximo crescimento em salinidade em torno de 7 (PARIDA et al., 2004). Rhizophora mucronata Lam. teve desenvolvimento máximo em salinidades variando de 8 a 18 (HOPPE-SPEER et al., 2011) em laboratório. Avicennia marina (Forsk.) Vierh., considerada mais tolerante à salinidade, apresenta crescimento ótimo em salinidade de 9 (CLOUGH, 1984; SPALDING et al., 2010).

Em geral, o mangue tem seu desenvolvimento ótimo em baixa salinidade. Contudo, algumas espécies toleram salinidade mais elevada do que outras (BALL, 1988). Espécies do gênero Rhizophora são mais sensíveis ao aumento de salinidade do que aquelas do gênero Avicennia (CLOUGH, 1984). As diferenças estão presentes dentro dos gêneros. Rhizophora apiculata Blume é mais sensível do que Rhizophora stylosa Griff. (BALL et al., 1997).

É notório que as espécies de manguezal são classificadas como vegetação halófita (TOMLINSON, 1986; PARIDA; JHA, 2010), pois crescem e se reproduzem na presença de sal. Alguns autores as classificam como halófitas facultativas, pois seu crescimento ótimo ocorre em baixa salinidade (TOMLINSON, 1986; PARIDA; JHA, 2010). Sustentase esta hipótese porque o aumento da salinidade causa desequilíbrios pelo excesso de íons $\mathrm{Na}^{+} \mathrm{e} \mathrm{Cl}^{-} \mathrm{em}$ diversos órgãos vegetais. As respostas aos distúrbios variam de espécie para espécie dependendo da tolerância.
Normalmente, manguezais diminuem o potencial hídrico dos seus tecidos com relação ao sedimento, permitindo com isto a absorção de água (TOMLINSON, 1986), sendo este o primeiro dos mecanismos de ajuste ao excesso de sais. À medida que a salinidade do substrato aumenta, ocorre efeito similar nas concentrações de $\mathrm{Na}^{+}$ e $\mathrm{Cl}^{-}$. Este fato não é acompanhado pela entrada de íons $\mathrm{K}^{+}$para espécies menos tolerantes à salinidade. Estudos verificaram aumento na razão $\mathrm{Na}^{+} / \mathrm{K}^{+}$para Bruguiera gymnorrhiza (L.) Lam. crescendo em 35 de salinidade. Entretanto, Avicennia marina mantém menor potencial hídrico por absorver seletivamente o $\mathrm{K}^{+}$(NAIDOO et al., 2002). Isto resulta em diversidade estrutural e funcional, levando em algumas situações ao estabelecimento de zonas como proposto por Davis Jr. (1940).

$\mathrm{O}$ acúmulo de íons leva à consequências como a desestabilização das membranas celulares além de induzirem estresse oxidativo por meio da formação de espécies reativas de oxigênio (PRISCO; GOMESFILHO, 2010). Experimentos realizados por Parida et al. (2003), com plântulas de Bruguiera parviflora em diferentes salinidades, apontaram que em 28 de salinidade há perda parcial do empilhamento granal, do citocromo B6f e dissociação de polipeptídios ligados intrinsecamente ao fotossistema II (FSII). Essas disfunções afetam diretamente a aquisição de carbono e, indiretamente, o incremento em biomassa. Além disso, hipersalinidade pode causar desintegração de cloroplastos e de mitocôndrias, como observado em $A$. marina submetida a 58 de salinidade (NAIDOO et al., 2011).

Além destes efeitos, o excesso de sal pode alterar a concentração de clorofila, o que pode reduzir a capacidade fotossintética (NAIDOO et al., 2002). No entanto, Sobrado (2005) observou aumento na concentração de clorofila $a$ e $b$ em Laguncularia racemosa, o que indica tolerância às condições impostas pela concentração de sais que, no experimento, não foram superiores a 30 . Normalmente, o aumento da salinidade vem acompanhado pela redução na concentração de clorofila devido à fotoinibição crônica, pois o teor salino é superior ao tolerado para a espécie estudada, como visto nos trabalhos de Sobrado (1999), Naidoo et al. (2002; 2011) e Parida et al. (2003). 
Todos estes fatores associados à desativação do FSII levam à diminuição da atividade fotoquímica. A redução da eficiência fotoquímica $\left(\mathrm{F}_{\mathrm{v}} / \mathrm{F}_{\mathrm{m}}\right)$ com incremento em salinidade é observada por vários autores para A. marina, B.parviflora e R. mucronata (PARIDA et al., 2003; HOPPE-SPEER et al., 2011; NAIDOO et al., 2011). O estresse salino provoca também redução da condutância estomática e da condução no mesofilo, limitando a fotossíntese por baixa difusão do $\mathrm{CO}_{2}$ (PARIDA et al., 2004), além de diminuir condutividade hidráulica provocando o fechamento dos estômatos (SOBRADO, 2001).

\section{Estratégias de absorção de água em condições salinas}

Mesmo crescendo em um ambiente inundado, a salinidade elevada dificulta a absorção de água pelas plantas (PALIYAVUTH et al., 2004). Assim, as espécies de mangue têm mecanismos para absorver a água contra fortes potenciais osmóticos (TOMLINSON, 1986). Crescer em um meio salino cria basicamente dois problemas para a planta: (1) a regulação osmótica e (2) a toxidade destes íons. Assim, as plantas têm de absorver os íons inorgânicos para neutralizar o gradiente osmótico. Todavia, o excesso destes no interior da célula torna-se tóxico e tem efeitos negativos sobre a reprodução, o crescimento e sobrevivência das plantas (MEHLIG, 2006).

Para manter a absorção de água, as plantas de mangue não apenas precisam restringir a perda de água por meio de adaptações morfológicas e fisiológicas (espessamento foliar, aumento do tempo de retenção do nitrogênio e eficiência no uso da água), mas também manter os potenciais de água suficientemente baixos (LOVELOCK; FELLER, 2003; KRAUSS et al., 2008) no interior das folhas. Estes aspectos fazem com que a eficiência de uso da água, em algumas das espécies de mangue, seja considerada uma das mais altas de todas as plantas $\mathrm{C}_{3}$ (BALL, 1986), uma vez que estas normalmente apresentam baixas taxas de transpiração e condutância estomática (BALL; FARQUHAR, 1984a; 1984b; BALL, 1988; LOVELOCK; BALL, 2002; SOBRADO, 2005).
Entretanto, apesar dessas estratégias, para compensar a elevada osmolaridade de sal no solo, um dos mecanismos bioquímicos empregados é o acúmulo de solutos compatíveis, a partir do aumento de moléculas pequenas de osmólitos como a glicina-betaína ou álcoois de açúcar (POPP et al., 1985; SAKAMOTO; MURATA, 2000) que ampliam o potencial osmótico sem alterar o iônico. Reduções nos potenciais osmóticos atraem água para dentro da célula e permitem manter o potencial de pressão (MOGHAIEB et al., 2004) necessário para obter água.

A melhor eficiência do uso da água associada aos custos energéticos de excretar e/ou excluir os sais é à custa de taxas reduzidas de assimilação de carbono (BALL, 1988). Quanto maior o teor de sal no local menor será a produtividade e isto irá refletir na estrutura do bosque.

Durante os períodos de elevada demanda evaporativa, plantas que excluem sal precisam economizar água por causa da capacidade limitada para extraí-la dos solos salinos (BARR et al., 2009). Smith et al. (1989) mostraram que a razão para a redução da taxa de assimilação de carbono do mangue na Venezuela foi em parte devido à salinidade elevada, o que causou maior potencial osmótico no xilema e redução da condutância estomática. Em ambientes hipersalinos, os manguezais apresentam diminutas taxas de assimilação de carbono e condutância estomática, originando bosques descritos como anões (LUGO; SNEDAKER, 1974).

Outro problema enfrentado pelos manguezais, devido à salinidade e a elevada demanda evaporativa, é sua vulnerabilidade à embolia ou cavitação que pode obstruir a condutância hidráulica. Essa vulnerabilidade é, principalmente, presente em ambientes hipersalinos, pois apresentam dificuldade em absorção de água (LOVELLOCK et al., 2006a; 2006b; MARTIN et al., 2010). Estudos de Sobrado (2001), com Avicennia germinans, demonstram que as espécies com maior eficiência no uso da água possuem esta capacidade devido à redução na condução de seiva no caule. Esta estratégia é possível porque as espécies reduzem a condutância estomática a ponto de impedir a embolia nas células do xilema. 
Tomlinson (1986), ao descrever o tecido xilemático do mangue, já associa como mecanismos para evitar a embolia a diminuição no diâmetro celular. Hao et al. (2009), comparando a pressão osmótica no solo sob diferentes salinidades em períodos diurnos e noturnos para Rhizophora mangle, identificam que a redistribuição da seiva bruta ou reversão da condução ocorre para evitar a embolia, além de amenizar a salinidade no sedimento em situações de baixa frequência de inundação.

\section{Mecanismos de tolerância à salinidade}

As estratégias para determinar baixas taxas de perda de água foliar também limitam o ganho de carbono, fazendo com que espécies que têm ampla tolerância ao sal cresçam mais lentamente com aumento da salinidade do que aquelas menos tolerantes (BALL, 1986; 1988).

De acordo com suas características, as espécies de mangue podem ser classificadas em secretoras de sal aquelas que têm glândulas de sal ou pelos para remover o sal - e não secretoras - que não apresentam mecanismos para a excreção do sal (SCHOLANDER et al., 1962; TOMLINSON, 1986). Larcher (2006) descreve as halófitas como plantas que toleram ou que resistem ao sal. Aquelas que resistem ao sal apresentam mecanismos fisiológicos que são: evitar a entrada, diluir e eliminar o sal. As classificadas como tolerantes seriam aquelas que toleram o sal no citoplasma. De acordo com Salisbury e Ross (1994), estas seriam as halófitas verdadeiras (ou euhalófitas). Somente as tolerantes seriam capazes de tolerar os efeitos tóxicos do cloro e sódio no citoplasma (LARCHER, 2006; SALISBURY; ROSS, 1994).

Popp et al. (1993), revisando os conceitos propostos por Scholander (1968) e a classificação de Larcher (2006), avaliam a capacidade da vegetação de mangue como capaz de evitar a entrada de sais via raiz e as estratégias de extrusão e/ou compartimentação dos sais. Concluem que os entendimentos sobre os processos de exclusão do sódio e cloro antes do ingresso no xilema não são totalmente entendidos, havendo espécies que não demonstram diferenças entre as pressões osmóticas do sedimento, das raízes, dos caules e das folhas como afirmado por Scholander (1968). Por outro lado, afirmam que as espécies que promovem a extrusão do sal via glândulas são reduzidas dentro da gama de espécies de mangue.

Em relação à compartimentação dos sais, este fenômeno é descrito sendo comum entre as espécies de mangue, já que várias apresentam maior conteúdo de sais nas folhas senescentes quando comparadas às novas (POPP et al., 1993). Além disso, estudos realizados pelos autores revelaram que com o aumento da salinidade ocorre acréscimo de metabólitos secundários, como o manitol, no citoplasma, concomitante ao incremento de sódio e cloro nos vacúolos.

Os gêneros Aegiceras, Avicennia, Acanthus e Aegialitis têm estruturas glandulares de excreção do sal enquanto Laguncularia e Conocarpus têm estrutura análoga às glândulas de sal (TOMLINSON, 1986). Bruguiera gymnorrhiza e Rhizophora mucronata também excluem sal, no entanto, num processo definido como realocação dos sais para partes senescentes, onde o sal é acumulado nas folhas mais maduras, as quais são eliminadas para excluir o excesso (STEINKE, 1999). Diferentes espécies têm distintas faixas de tolerância ao sal (SUAREZ; MEDINA, 2005) correlacionadas a sua capacidade de excreção e/ou eliminação dos sais. Aquelas que excretam os sais diariamente possuem capacidade de colonizar ambientes com salinidades mais elevadas, ao contrário daquelas que armazenam os sais por períodos maiores (por compartimentação ou realocação) só eliminando-os com morte de suas estruturas vegetais.

Avicennia são conhecidas por excluírem cerca de $90 \%$ de sal absorvidos a partir da superfície da raiz, que pode aumentar à medida que a concentração de sal da água se amplia (BRANCH; BRANCH, 1995). A exclusão de sal é obtida criando-se pressão hidrostática negativa na célula que fornece pressão osmótica suficientemente negativa para as raízes para absorção de água, enquanto todos os outros íons são excluídos (MOON et al., 1986). Estes autores avaliaram que, além do processo de ultrafiltração, a extrusão do sal via sistema de raízes para as espécies de Avicennia é controlada por aspectos anatômicos que inviabilizam o transporte apoplástico da água até o xilema. Este transporte é impedido pela suberização das células do córtex da raiz e também pela banda de Caspary da endoderme. Suarez e Medina 
(2005) mostraram que plântulas de $A$. geminans foram significativamente afetadas pela salinidade e tiveram tolerância ao sal elevada em comparação com outras espécies semelhantes, como Aegiceras corniculatum (L.) Blanco e Avicennia marina.

Estudo com Bruguiera gymnorhiza indicou que área, massa foliar por planta e longevidade aumentaram com o incremento da salinidade (WANG; LIN, 1999). A suculência foliar pode ser um mecanismo para evitar concentrações de sal excessivamente elevadas (HAGEMEYER, 1997), uma vez que o aumento do volume do vacúolo permite a diluição dos íons solúveis.

Redução do crescimento (menor altura, diminuição da biomassa e produção de folha) pode ser interpretada como resultado do investimento em mecanismos de tolerância e/ou resistência ao sal (SNEDAKER, 1982; SUAREZ; MEDINA, 2005), já que a manutenção de tais estratégias reduz a produtividade primária líquida.

Segundo Kathiresan e Thangam (1990), as flutuações na salinidade dentro do ambiente parecem ter efeitos mais pronunciados do que a hipersalinidade contínua, uma vez que as plantas adultas têm faixa superior de tolerância em relação às plântulas. Isso acontece porque as plantas mais jovens são geralmente mais sensíveis ao sal, crescendo melhor em locais onde as salinidades são menores e, porteriormente, tornam-se mais tolerante (SCHMITZ et al., 2006). Entretanto, uma vez o bosque estabelecido, modificações na salinidade podem levar ao colapso do indivíduo adulto por menor plasticidade que as plântulas, como observado em situações onde houve alterações na dinâmica de inundação provocadas por modificações na desembocadura do rio (TOGNELLA et al., 2007). Os autores observaram que as alterações na salinidade tiveram como resposta da vegetação a morte das folhas na porção mais alta da copa. Relacionaram tal fato às alterações do potencial osmótico que podem ter ocorrido nos ramos terminais inviabilizando a manutenção das folhas por dificuldade no aporte de água. Com isto, os indivíduos adultos tiveram sua estatura diminuída, permitindo sua sobrevivência.

\section{Inundação e alagamento}

As florestas de manguezal são regularmente lavadas pelas marés. A circulação da água elimina substâncias tóxicas para a vegetação, como o sulfeto de hidrogênio $\left(\mathrm{H}_{2} \mathrm{~S}\right)$ e oxigenam o sedimento (KNIGHT et al., 2008). Os padrões de inundação das florestas são diferenciados e refletem na estrutura da mesma. Os bosques de franja são constantemente inundados pelo movimento da maré ventilando e removendo os compostos tóxicos e os bosques de bacia são geralmente inundados nas marés mais altas (SCHAEFFER-NOVELLI et al., 2000; KNIGHT et al., 2008) ficando matéria orgânica em decomposição por período maior, causando, em algumas situações, condições de anoxia para as raízes.

Em condições de alagamento, o mangue deve lidar com parcial ou completa ausência de oxigênio dissolvido para respiração das raízes (MCKEE, 1996; SKELTON; ALLAWAY, 1996). Essas espécies, que caracterizam a vegetação de mangue, diferem em sua capacidade de manter o tecido da raiz aerado afetando a fisiologia e morfologia das raízes (MCKEE, 1996). A hipoxia causa diminuição na taxa de extensão da raiz em Avicennia germinans e Laguncularia racemosa, mas não em Rhizophora mangle. Além disso, a hipoxia é responsável pela morte de raízes de L. racemosa e pela diminuição no número de raízes laterais nas três espécies neotropicais citadas acima e, também, pelo aumento da parte aérea em A. germinans e L. racemosa (MCKEE, 1996). Bruguiera mucronata apresentou similar tendência, com maior altura quando submetida à inundação constante, revelando mudança na alocação de biomassa para a parte aérea (HOPPE-SPEER et al., 2011).

Estudo realizado com plântulas de Avicennia marina apontou que o estresse causado apenas por inundação pode não ser tão prejudicial à espécie (SKELTON; ALLAWAY, 1996). As plântulas de A. marina em condições de maré baixa aumentam a concentração de oxigênio em suas raízes até concentração estável. Quando são submetidas à inundação por seis e 22 horas, a concentração de oxigênio nos pneumatóforos e raízes de suporte diminui significativamente, principalmente, quando em maior tempo de inundação. Porém, a concentração restante de oxigênio é suficiente 
para manter metabolismo aeróbico no nível de raiz (SKELTON; ALLAWAY, 1996). Essa concentração é determinada pela capacidade que as raízes têm de armazenar oxigênio na maré baixa, que pode estar ligada ao aerênquima nas raízes, pois, em maré alta, o espaço interno de gás está isolado do exterior e há transferência de oxigênio da estrutura aérea às raízes (TOMLINSON, 1986; SKELTON; ALLAWAY, 1996).

Estudos em laboratório com plântulas mostraram que as inundações em longo prazo não têm efeito persistente sobre as trocas gasosas em Avicennia germinans, Laguncularia racemosa ou Rhizophora mangle (PEZESHKI et al., 1990; KRAUSS et al., 2006). Já a inundação de curto prazo reduz a assimilação máxima e respiração no escuro em plântulas de $A$. germinans, $L$. racemosa e $R$. mangle de acordo com os estudos de Krauss et al. (2006). No entanto, os mangues maduros podem ser menos sensíveis à inundação de curto prazo do que as plântulas (NAIDOO et al., 1998). Apesar dos aumentos da concentração do $\mathrm{CO}_{2}$ atmosférico resultarem em taxas de crescimento elevadas, estas são menores do que as reduções nas taxas de crescimento observadas quando manguezais estão cada vez mais inundados (FARNSWORTH et al., 1995).

\section{Nutrientes}

O input de nutrientes no ecossistema manguezal é diferenciado nos bosques de franja e de bacia. Os bosques de franja são expostos a duas condições nutricionais dependendo da sua localização, próximo ao mar ou ao rio. Basicamente, os bosques de franja próximos ao mar são oligotróficos, pois recebem menor descarga de nutrientes a partir dos rios. Por outro lado, os bosques de franja no interior dos estuários recebem grande aporte de nutrientes provenientes do fluxo fluvial. Os bosques de bacia requerem input de nutrientes a partir das chuvas e do escoamento terrestre para melhor desenvolvimento, pois são inundados apenas nas marés altas. As bacias desenvolvem-se exuberantemente nos locais onde a precipitação é maior do que a evapotranspiração local, isto é, não ocorrem estações com déficit hídrico. Se a evapotranspiração é maior do que a precipitação esses bosques tem crescimento reduzido, pois além de lidar com déficit nutricional suportam também as condições de elevada salinidade ou até mesmo hipersalinidade (SCHAEFFER-NOVELLI et al., 2000) que, em algumas situações, podem resultar em áreas de apicum, que são regiões hipersalinas sem vegetação.

Muitas áreas de manguezal em locais diferentes do globo sofrem com a reduzida disponibilidade de nutrientes, principalmente nitrogênio e fósforo, sendo estes os macroelementos de maior limitação ao crescimento da vegetação (ALONGI, 2011). Normalmente essas áreas são bosques de bacia permanentemente alagados, com a renovação de água só ocorrendo nas marés mais extremas do ano. Além disso, apresentam salinidade acima daquela da água do mar. Juntamente ao reduzido fornecimento de nutrientes, processos microbianos, como a taxa de decomposição e retenção de nutrientes na rizosfera, podem reduzir a disponibilidade de nutrientes para a absorção pelas plantas (NAIDOO, 2006).

Deficiência de $\mathrm{P}$ está extremamente ligada à diminuição de transporte de água, pois diminui o funcionamento de canais de água (aquaporinas), que necessitam de fosforilação para o funcionamento (LOVELOCK et al., 2006a). Essa deficiência leva à redução da condutância estomática e condutividade hidráulica, diminuição da ramificação da parte aérea e alongamento celular, reduzindo o crescimento das árvores. Portanto, em locais limitados por $\mathrm{P}$ os processos de coleta e transporte de água são pontos chave para restringir a produtividade nesses bosques (LOVELOCK et al., 2004; 2006a; 2006b; NAIDOO, 2006).

A deficiência em $\mathrm{N}$ também inibe o crescimento em altura das árvores. Esse elemento é utilizado na incorporação de biomassa (compõem proteínas), produção de solutos compatíveis e participam na composição de RUBISCO (enzima responsável pela redução do $\mathrm{CO}_{2}$ ) (POOP et al., 1985; MARTIN et al., 2010). Normalmente, indivíduos com deficiência em $\mathrm{N}$ apresentam menor área foliar, menor eficiência fotoquímica e taxa fotossintética. Bosques limitados por $\mathrm{N}$, quando fertilizados, têm sua melhoria em produção primária quando mais carbono é alocado na área foliar, que pode levar, em alguns casos, ao aumento em taxa fotossintética (LOVELOCK; FELLER, 2003; LOVELOCK et al., 2006a; MARTIN et al., 2010). 
Em experimentos de longo prazo, demonstrado por Feller (1995) em comunidades crescendo sobre solos calcários em Twin Cays Belize, a disponibilidade de $\mathrm{P}$ foi o fator ecológico determinante para o crescimento das plantas. Medina et al. (2010) também associaram o crescimento deficiente de $R$. mangle, no leste de Porto Rico, com uma combinação de limitação de $\mathrm{P}$ e estresse hídrico sazonal. Lovelock et al. (2006c), ao estudar manguezais onde $\mathrm{P}$ era o nutriente limitante, a fertilização com P resultou num aumento do crescimento, na condutância hidraúlica do caule, na transpiração e na fotossíntese. Já estudos no Indo-Pacífico e no Continente Africano (LOVELOCK et al., 2007) mostraram variação no crescimento independente de ser o $\mathrm{N}$ ou $\mathrm{P}$ os fatores limitantes, sendo $\mathrm{N}$ aquele nutriente que mais frequentemente limitou o crescimento das plantas. No manguezal onde o $\mathrm{N}$ era limitante, a fertilização melhorou o crescimento do dossel, mas sem alterar a condutividade hidraúlica específica dos caules e das folhas (LOVELOCK et al., 2006b).

Estudando espécies de Avicennia gernimans crescendo em dois ambientes com salinidades contrastantes na Australia, Martin et al. (2010) não encontraram efeito significativo da fertilização com $\mathrm{P}$ no crescimento das partes aéreas, enquanto a fertilização com $\mathrm{N}$ melhorou o desenvolvimento do dossel, aumentando a quantidade de folhas, particularmente das árvores crescendo na área hipersalina. Assim, segundo estes autores, parece que as restrições sobre a absorção de água em um ambiente salino pode ser superada, em certa medida, com a fertilização de $\mathrm{N}$, aumentando o investimento do $\mathrm{N}$ na Rubisco e uma redução no custo hídrico do ganho de carbono em nível foliar. Lovelock e Feller (2003), ao avaliarem a fertilização de $A$. germinans versus Laguncularia racemosa constataram que o aumento do desempenho fotossintético em $A$. germinans com $\mathrm{N}$ era muito maior do que o de $L$. racemosa fertilizado com o mesmo nutriente.

$\mathrm{O}$ nutriente Fosfato $\left(\mathrm{PO}_{4}^{3-}\right)$ em solos de mangue pode ser imobilizado e indisponível para uso da planta. Assim, os organismos que solubilizam $\mathrm{P}$ podem ter implicações importantes para o crescimento das plantas, especialmente em ambientes onde os nutrientes são limitados (REEF et al., 2010).

\section{Mudanças climáticas}

Os ecossistemas costeiros, como o manguezal, são extremamente susceptíveis às alterações climáticas (ALONGI, 2008). Elas podem afetar tanto a planta quanto os processos bioquímicos do solo, pelo aumento dos níveis de $\mathrm{CO}_{2}$, temperatura, nível médio do mar e maior frequência de tempestades. Estes são todos aspectos possíveis que podem causar um impacto significativo sobre a fisiologia do mangue e sobre o funcionamento do ecossistema (REEF et al., 2010).

Aumento da concentração de $\mathrm{CO}_{2}$ atmosférico pode causar aumento em assimilação fotossintética e crescimento das plantas de mangue em baixas salinidades, como observado em plântulas de Rhizophora apiculata e Rhizophora stylosa cultivadas em laboratório (BALL et al., 1997). No entanto, diferenças interespecíficas em resposta ao aumento de $\mathrm{CO}_{2}$ e sua relação às outras mudanças climáticas podem alterar padrões de zonação dentro de estuário (ALONGI, 2008).

Alongi (2008) propõe que o aumento da temperatura atmosférica, além de expandir os limites latitudinais do ecossistema, diminuiria a assimilação fotossintética em áreas áridas e aumentaria em áreas de elevada pluviosidade. Em adição, o aumento de temperatura pode levar a mudanças na composição da comunidade devido às diferenças entre as espécies em manter a temperatura foliar alguns graus abaixo daquela do ar (LUGO et al., 2007). Barr et al. (2009) estudando indivíduos de Rhizophora mangle na Flórida, verificaram que em situações onde a temperatura do ar excedeu os $30^{\circ} \mathrm{C}$ houve desativação fotossintética induzida pelo incremento na temperatura, o que contribuiu para reduzir a taxa de assimilação de carbono.

Em relação ao aumento no nível do mar, Mcfadden et al. (2007) sugerem que cerca de 33 a $44 \%$ dos manguezais mundiais podem ser perdidos no período de 2000 a 2080, devido ao aumento em torno de 36 a $72 \mathrm{~cm}$ do nível médio relativo do mar. No entanto, áreas de manguezal podem sobreviver ao aumento do nível do mar dependendo da taxa de acúmulo de sedimento em relação à taxa de mudança do nível do mar, como ressaltado por Alongi (2008) e Soares (2009). Além disso, manguezais com presença de áreas de apicum têm 
a possibilidade de migrar em direção a essas planícies, como observado em Guaratiba, Brasil (SOARES, 2009).

Do ponto de vista fisiológico, o aumento do nível do mar diminui a altura e taxa de crescimento dos indivíduos (FARNSWORTH et al., 1995), além de resultar em mudanças na composição da comunidade devido as diferenças na tolerância ao alagamento entre as espécies (ALONGI, 2008).

Aparentemente, alterações ambientais isoladas, como o aumento na concentração de $\mathrm{CO}_{2}$, por exemplo, podem induzir respostas positivas do manguezal. Porém, quando estes fatores estiverem associados à elevação no nível do mar e ocorrendo em velocidade acentuada, as respostas da vegetação poderão ser negativas.

As respostas do manguezal às mudanças climáticas serão diversas conforme relatado acima, entretanto, assume-se que estes poderão se expandir latitudinalmente (SOARES et al., 2012) desde que haja áreas disponíveis para isto. Localmente, haverá uma expansão em direção ao continente em áreas onde hoje ocorrem apicuns ou sobre outros ecossistemas. Áreas urbanizadas inviabilizarão a expansão dos manguezais retroterra.

Por outro lado, a elevação da temperatura poderá acarretar a competição das espécies de mangue com plantas de marismas que tem fisiologia $\mathrm{C}_{4}$ e com isto assimilam $\mathrm{CO}_{2}$ de forma mais eficiente em temperaturas acima de $40^{\circ} \mathrm{C}$ (ADAM, 1990).

Concluindo, esta análise reporta que os estudos ecofisiológicos da vegetação de mangue são pontuais, concentrados em espécies da região do Indo Pacífico e, em alguns, apresentam resultados distintos entre experimentos in situ e em condições de laboratório. Ambientes onde ocorre maior diversidade específica indicam maior resiliência para as mudanças climáticas. No Brasil, o conhecimento das questões de ecofisiologia do manguezal ainda são incipientes sendo urgente a elaboração de estudos que contribuam para o entendimento dos processos fisiológicos desta vegetação e que permitam o gerenciamento adequado haja vista as mudanças climáticas esperadas. Recomendamos que sejam envidados esforços para melhor entendimento dos processos de assimilação de carbono e de nutrientes em condições naturais.

\section{Agradecimentos}

Os autores agradecem o apoio do Instituto Estadual de Meio Ambiente (IEMA) e ao Gerente Ambiental da APA de Conceição da Barra e ao Mosaico de Manguezais de Vitória onde as pesquisas de ecofisiologia vegetal estão sendo realizadas e permitiram a elaboração desta síntese. A primeira autora agradece a Pró-Reitoria de Pesquisa e Pós-Graduação da UFES (Universidade Federal do Espírito Santo) pelos dois anos de bolsa de Iniciação Científica e FAPES (Fundação de Apoio à Pesquisa e Inovação do Espírito Santo) pela atual bolsa de mestrado. A segunda autora agradece a CAPES pela bolsa de mestrado. A última autora agradece à FAPES pela bolsa pesquisador capixaba. As pesquisas de fluorescência têm subsídio financeiro dos projetos de pesquisa PELD (CNPq) - Processo $\mathrm{N}^{\mathrm{o}} 558246 / 2009-5$ e Universal (CNPq) - Processo No 483364/2010-0.

\section{Referências}

ADAM, P. Salt Marsh Ecology. London: Cambridge University Press, 1990. 286 p.

ALONGI, D M. Mangrove forests: Resilience, protection from tsunamis, and responses to global climate change. Estuarine, Coastal, and Shelf Science, Townsville, v. 76, p. 1-13, 2008.

ALONGI, D M. Early growth responses of mangrove to different rates of nitrogen and phosphorus supply. Journal of Experimental Marine Biology and Ecology, Philadelphia, v. 397, p. 85-93, 2011. BALL, M. C. Photosynthesis in mangroves. Wetlands, Madison, v. 6, n. 1, p. 12-22, 1986.

BALL, M. C. Ecophysiology of mangroves. Trees, New York, v. 2, p. 129-142, 1988.

BALL, M. C.; COCHRANE, M. J.; RAWSON, H. M. Grow and water use of the mangroves Rhizophora apiculata and $R$. stylosa in response to salinity and humidity under ambient and elevated concentrations of atmospheric $\mathrm{CO}_{2}$. Plant, Cell and Environment, Malden, v. 20, p. 1158-1166, 1997.

BALL, M. C.; FARQUHAR, G. F. Photosynthetic and stomatal responses of the grey mangrove, Avicennia marina, to transient salinity conditions. Plant Physiology, Rockville, v. 74, p. 7-11, 1984a.

BALL M. C.; FARQUHAR G. D. Photosynthetic and stomatal responses of 2 mangrove species, Aegiceras corniculatum and Avicennia marina, to long-term salinity and humidity conditions. Plant Physiology, Rockville, v. 74, p. 1-6, 1984b.

BARR, J. G.; FUENTES, J. D.; ENGEL, V.; ZIEMAN, J. C. Physiological responses of red mangroves to the climate in the Florida Everglades. Journal of Geophysical Research, Malden, v. 114, p. 1-13, 2009. 
BRANCH, M.; BRANCH, G. The living shores of Southern Africa. Cape Town: Struik Publishers, 1995. 272 p.

CLOUGH, B. F. Growth and salt balance of the mangroves Avicennia marina (Forsk.) Vierh. and Rhizophora stylosa Griff. in relation to salinity. Australian Journal of Plant Physiology, Collingwood, v. 11, n. 5, p. 419-430, 1984.

DAVIS JR, J. H. The ecology and geologic role of mangroves in Florida. Papua Tortugus Laboratory. Carnegie Institute, Washington, v. 32, p. 304-412, 1940.

FARNSWORTH, E. The ecology and physiology of viviparous and recalcitrant seeds. Annual Review of Ecology and Systematics, Palo Alto, v. 31, p. 107-138, 2000.

FARNSWORTH, E.; ELLISON, A. M.; GONG, W. K.; BAZZAZ, F. A. Ecophysiological responses of mangrove seedlings to two facets of climate change. Bulletin of the Ecological Society of America, New York, v. 77, p. 76-88, 1995.

FELLER, I. C. Effects of nutrient enrichment on growth and herbivory of dwarf red mangrove (Rhizophora mangle). Ecological Monographs, New York, v. 65, p. 477-505, 1995.

HAO, G-Y; JONES, T. J.; LUTON, Z.; ZHANG, Y-C; MANZONE, E.; SCHOLZ, F. Z.; BUCCI, S. J; CAO, K-F; GOLDSTEIN, F. Hydrolic redistribution in dwarf Rhizophora mangle tree driven by interstitial soil water salinity gradients: impacts on hydraulic architecture and gas exchange. Tree Physiology, Victoria, v. 29, p. 697-705, 2009.

HAGEMEYER, J. Salt. In: PRASAD, M. N. V. (Ed.). Plant ecophysiology. New York: Wiley, 1997. p. 173-206.

HOPPE-SPEER, C. L.; ADAMS, J. B.; RAJKARAN, A.; BAILEY, D. The response of the red mangrove Rhizophora mucronata Lam. to salinity and inundation in South Africa. Aquatic Botany, Amsterdam, v. 95, p. 71-76, 2011.

KATHIRESAN, K.; THANGAM, T. S. Effect of phenolics on growth of viviparous seedlings of Rhizophora apiculata Blume. Geobios, Amsterdam, v. 17, p. 142-143, 1990.

KNIGHT, J. M.; DALE, P. E. R.; DUNN, R. J. K.; BROADBENT, G. J.; LEMCKERT, C. J. Patterns of tidal flooding within a mangrove forest: Coombabah Lake, Southeast Queensland, Australia. Estuarine, Coastal and Shelf Science, Townsville, v. 76, n. 3, p. 580-593, 2008.

KRAUSS, K. W.; KEELAND, B. D.; ALLEN, J. A.; EWEL, K. C.; JOHNSON, D. J. Effects of season, rainfall, and hydrogeomorphic setting on mangrove tree growth in Micronesia. Biotropica, Malden, v. 39, p. 161-170, 2006.

KRAUSS, K. W.; LOVELOCK, C. E.; MCKEE, K. L.; LÓPEZHOFFMAN, L.; EWE, S. M. L.; SOUSA, W. P. Environmental drivers in mangrove establishment and early development: a review. Aquatic Botany, Amsterdam, v. 89, p. 105-127, 2008.

LARCHER, W. Ecofisiologia vegetal. São Carlos: RiMa, 2006. 531 p. LIBES, S. M. Introduction to marine biogeochemistry. Amsterdam: Academic Press, 2009. 909 p.

LOVELOCK, C. E.; BALL, M. C. Influence of salinity on photosynthesis of halophytes. In: LAUCHLI, A.; LUTTAGE, U. (Ed.). Salinity: environment - plants - molecules. New York: Kluwer Academic Publishers, 2002. p. 315-339.

LOVELOCK, C. E.; BALL, M. C.; CHOAT, B; ENGELBRECHT, B. M. J.; HOLBROOK, N. M.; FELLER, I. C. Linking physiological processes with mangrove forest structure: phosphorus deficiency limits canopy development, hydraulic conductivity and photosynthetic carbon gain in dwarf Rhizophora mangle. Plant, Cell and Environment, Malden, v. 29, p. 793-802, 2006a.

LOVELOCK, C. E.; BALL, M. C.; FELLER, I. C.; ENGELBRECHT, B. M. J.; EWE, M. L. Variation in hydraulic conductivity of mangroves: influence of species, salinity, and nitrogen and phosphorus availability. Physiologia Plantarum, Malden, v. 127, p. 457-464, 2006 b.

LOVELOCK, C. E.; FELLER, I. C. Photosynthetic performance and resource utilization of two mangrove species coexisting in a hypersaline scrub forest. Oecologia, New York, v. 134, p. 455-462, 2003.

LOVELOCK, C. E.; FELLER, I. C.; BALL, M. C.; ELLIS, J.; SORRELL, B. Testing the growth rate vs. geochemical hypothesis for latitudinal variation in plant nutrients. Ecology Letters, Malden, v. 10, p. 1154-1163, 2007.

LOVELOCK, C. E.; FELLER, I. C.; MCKEE, K. L.; ENGELBRECHT, B. M. J.; BALL, M. C. The effect of nutrient enrichment on growth photosynthesis and hydraulic conductance of dwarf mangroves in Panamá. Functional Ecology, London, v. 18, p. 25-33, 2004.

LOVELOCK, C. E.; RUESS, R. W.; FELLER, I. C. Fine root respiration in the mangrove Rhizophora mangle over variation in forest stature and nutrient availability. Tree Physiology, Oxford, v. 26, p. 1601-1606, 2006c.

LUGO, A. E.; MEDINA, E.; CUEVAS, E.; CINTRON, G.; NIEVES, E. N. L.; SCHAEFFER-NOVELLI, Y. Ecophysiology of a Mangrove Forest in Jobos Bay, Puerto Rico. Caribbean Journal of Science, Mayaguez, v. 43, n. 2, p. 200-219, 2007.

LUGO, A. E.; SNEDAKER S. C. The ecology of mangroves. Annual Review of Ecology and Systematics, Palo Alto, v. 5, p. 39-64, 1974. MARTIN, K. C.; BRUHN, D.; LOVELOCK, C. E.; FELLER, I. C.; EVANS, J. R.; BALL, M. C. Nitrogen fertilization enhances water-use efficiency in a saline environment. Plant, Cell and Environment, Malden, v. 33, p. 344-357, 2010.

MCFADDEN, L.; SPENCER, T.; NICHOLLS, R.J. Broad-scale modeling of coastal wetlands: what is required? Hydrobiologica, New York, v. 577, p. 5-15, 2007.

MCKEE, K. L. Growth and physiological responses of neotropical mangrove seedlings to root zone hypoxia. Tree Physiology, Oxford, v. 16, p. 883-889, 1996.

MEDINA, E.; CUEVAS, E.; LUGO, A. Nutrient relations of dwarf Rhizophora mangle L. mangroves on peat in eastern Puerto Rico. Plant Ecology, New York, v. 207, p. 13-24, 2010.

MEHLIG, U. Phenology of the red mangrove, Rhizophora mangle L., in the Caete Estuary, Para, equatorial Brazil. Aquatic Botany, Amsterdam, v. 84, p. 158-164, 2006.

MOGHAIEB, R. E. A.; SANEOKA, H.; FUJITA, K. Effect of salinity on osmotic adjustment, glycinebetaine accumulation and the betaine aldehyde dehydrogenase gene expression in two halophytic plants, Salicornia europaea and Suaeda maritime. Plant Science, Philadelphia, v. 166, p. 1345-1349, 2004.

MOON, G. J.; CLOUGH, B. F.; PETERSON, C. A.; ALLAWAY, W. G. Apoplastic and symplastic pathways in Avicennia marina (Forsk.) Vierh. roots revealed by fluorescent tracer dyers. Australian Journal of Plant Physiology, Collingwood, v. 13, p. 637-648, 1986. 
NAIDOO, G. Factors contributing to dwarfing in the mangrove Avicennia marina. Annals of Botany, Oxford, v. 97, p. 1095-1101, 2006.

NAIDOO, G.; HIRALAL, O.; NAIDOO Y. Hypersalinity effects on leaf ultrastructure and physiology in the mangrove Avicennia marina. Flora, Philadelphia, v. 206, p. 814-820, 2011.

NAIDOO, G.; ROGALLA, H.; VON WILlERT, D. J. Field measurements of gas exchange in Avicennia marina and Bruguiera gymnorrhiza. Mangroves Salt Marshes, New York, v. 2, p. 99107,1998

NAIDOO G.; TUFFERS A. V.; VON WILLERT D. J. Changes in gas exchange and chlorophyll fluorescence characteristics of two mangroves and a mangrove associate in response to salinity in the natural environment. Trees-Structure and Function, New York, v. 16, p. 140-146, 2002

PALIYAVUTH, C.; CLOUGH, B.; PATANAPONPAIBOON, P. Salt uptake and shoot water relations in mangroves. Aquatic Botany, Amsterdam, v. 78, p. 349-360, 2004.

PARIDA, A. K.; DAS, A. B.; MITTRA, B. Effects of $\mathrm{NaCl}$ stress on the structure, pigment complex, composition, and photosynthetic activity of mangrove Bruguiera parviflora chloroplasts. Photosynthetica, New York, v. 41, n. 2, p. 191-200, 2003.

PARIDA, A. K.; DAS, A. B.; MITTRA, B. Effects of salt on growth, ion accumulation, photosynthesis and leaf anatomy of the mangrove, Bruguiera parviflora. Trees, New York, v. 18, p. 167-174, 2004.

PARIDA, A. K.; JHA, B. Salt tolerance mechanisms in mangroves: a review. Trees, New York, v. 24, p. 199-217, 2010.

PEZESHKI, S. R.; DELAUNE, R. D.; PATRICK, W. H. JR. Differential response of selected mangroves to soil flooding and salinity: gas exchange and biomass partitioning. Canadian Journal of Forest Research, Ottawa, v. 20, p. 869-874, 1990.

POPP, M.; LARHER, F.; WEIGEL, P. Osmotic adaptation in Australian mangroves. Vegetatio, Perth, v. 61, p. 247-253, 1985.

POPP, M.; POLANIA, J; WEIPER, M. Physiological adaptations to diferent salinity levels in mangrove. In: H. LIETH; A. AL MASSON (Ed.): Towards the rational use of high salinity tolerant plants. Vol. 1. New York: Kluwer Academic Publishers, 1993. p. 217-224.

PRISCO, J. T.; GOMES-FILHO, E. Fisiologia e bioquímica do estresse salino em plantas. In: GHEYI, H. R.; DIAS, N. S.; LACERDA, C. F. (Ed.). Manejo da salinidade na agricultura: estudos básicos e aplicados. Fortaleza: INCTSal, 2010. p. 143-159.

REEF, R.; FELLER, C. I.; LOVELOCK, C. E. Nutrition of mangroves. Tree Physiology, Oxford, v. 30, p. 1148-1160, 2010.

SAKAMOTO, A.; MURATA, N. Genetic engineering of glycinebetaine synthes is inplants: current status and implication for enhancement of stress tolerance. Journal Experimental Botany, Oxford, v. 51, p. 81-88, 2000.

SALISBURY, F. B.; ROSS, C. W. Fisiologia vegetal. México: Grupo Editorial Iberoamética, 1994. 759 p.

SCHAEFFER-NOVELLI, Y.; CINTRÓN-MOLERO, G.; SOARES, M. L. G.; TOGNELLA-DE-ROSA, M. M. P. Brazilian mangroves. Aquatic Ecosystem Health and Management, Amsterdam, v. 3, p. 561-570, 2000.

SCHOLANDER, P. F. How mangrove desalinated water. Physiologia Plantarum, Malden, v. 21, n. 1, p. 251-261, 1968.

SCHOLANDER, P. F.; HAMMEL, H. T.; HEMMINGSEN, E. A.;
GAREY, W. Salt balance in mangroves. Plant Physiology, Oxford, v. 37, p. 722-729, 1962.

SCHMITZ, N.; VERHEYDEN, A.; BEECKMAN, H.; KAIRO, J. G.; KOEDAM, N. Influence of a salinity gradient on vessel characters of the mangrove species Rhizophora mucronata. Annals of Botany, Oxford, v. 98, p. 1321-1330, 2006.

SKELTON, N. J.; ALLAWAY, W. G. Oxygen and pressure changes measured in situ during flooding in roots of the grey mangrove Avicennia marina (Forssk.) Vierh. Aquatic Botany, Amsterdam, v. 54, p. 165-175, 1996.

SMITH, J. A. C.; POPP, M.; LUTTGE, U.; CRAM, W. J.; DIAZ, M.; GRIFFITHS, H.; LEE, H. S. J.; MEDINA, E.; SCHAFER, C.; STIMMEL, K. H.; THONKE, B. Ecophysiology of xerophytic and halophytic vegetation of a coastal alluvial plain in northern Venezuela. VI. Water relations and gas exchange of mangroves. New Phytologist, Lancaster, v. 111, p. 293-307, 1989.

SNEDAKER, S. C. Mangrove species zonation: why? In: SEN, D. N.; RAJPUROHIT, K. S. (Ed.). Tasks for vegetation science. Vol. 2. Contributions to the ecology of halophytes. Boston/Londres: Dr. W. Junk Publisherst, 1982. p. 111-125.

SOBRADO, M. A. Drought effects on photosynthesis of the mangrove, Avicennia germinans, under salinities. Trees, New York, v. 13, p. 125-130, 1999.

SOBRADO, M. A. Relation of water transport to leaf gas exchange properties in three mangrove species. Trees, New York, v. 14, p. 258-262, 2000.

SOBRADO, M. A. Hydraulic properties of a mangrove Avicennia germinans as affected by $\mathrm{NaCl}$. Biologia Plantarum, New York, v. 44(3), p. 435-438, 2001.

SOBRADO, M. A. Leaf characteristics and gas exchange of the mangrove Laguncularia racemosa as affected by salinity. Photosynthetica, New York, v. 43, n. 2, p. 217-221, 2005.

SOARES, M. L. G. A conceptual model for the responses of mangrove forests to sea level rise. Journal of Coastal Research, Fort Lauderdale, v. 56, p. 267-271, 2009.

SOARES, M. L. G.; ESTRADA, G. C. D.; FERNANDEZ, V.; TOGNELLA, M. M. P. Southern limit of the Western South Atlantic Mangrove: assessment of the potential effects of global warming from a biogeographical perspective. Estuarine, Coastal and Shelf Science, Townsville, v. 101, p. 44-53, 2012.

SPALING, M.; KAINUMA, M; COLLINS, L. Word atlas of mangroves. 2 ed. London: Earthscan, 2010. 325 p.

STEINKE, T. Mangroves in South African estuaries. In: ALLANSON, B. R.; BAIRD, D. (Ed.). Estuaries of South Africa. Cambridge: Cambridge University Press, 1999. p. 119-140.

SUAREZ, N.; MEDINA, N. Salinity effect on plant growth and leaf demography of the mangrove, Avicennia germinans L. Trees, New York, v. 19, p. 721-727, 2005.

TOGNELlA, M. M. P.; SOARES, M. L. G.; ESPINOZA, H. C. F. Abordagens ecológicas em manguezal. In: TAVARES, L. F. M. (Ed.). Conservação dos ecossistemas costeiros do Espírito Santo. Vitória: Editora da UFES, 2007. p. 50-75.

TOMLINSON, P. B. The botany of mangroves. Cambridge: Cambridge University Press, 1986. 419 p.

WANG, W.; LIN, P. Influence of substrate salinity on the growth of mangrove species of Bruguiera gymnorrhiza seedling. Journal of Xiamen University, Xiamen, v. 38, p. 273-279, 1999. 